\title{
Improving the management and mobility of BJPP
}

A $t$ the time when the third issue of volume 24 of BJPP is being launched, some remarks are to be commented by the Editorial Office.

First of all, the editorial committee of BJPP is working hard so that the journal can be published on time. Therefore, Associate Editors and reviewers are being encouraged to receive and process the peer review as quickly as possible. In 2012, the flow of manuscripts has been our major priority. When the text gets to our inbox, I give the manuscripts a first read and reject ones that are not appropriate for our journal, as some points as as purpose, originality and quality are checked quickly. Once the MS receives an initial approval, we commence a delicate task of finding scholars with sufficient expertise to review each one of the manuscripts. That is a big challenge for us, from the Editorial Office of BJPP. We tend not to publish manuscripts that simply apply experiments like dose-response to real problems in plants or in plant communities. Then, the manuscript is assigned to an associate editor and at least two reviewers, who should return it to the Editor-in-Chief in 60 days, at most. After this period, the Editor-in-Chief organizes the comments made by the reviewers and associate editors, and establishes his own opinion on the integrity of the manuscript. Since the article is returned to the author in up to ten days, as long as considering all the suggestions, the article is finally accepted for publication. At the end, the time predicted from submission to acceptance is of up to 75 days. In real numbers, we still have not reached this predicted time until now. The editorial committee of BJPP is making efforts to reach this goal in 2013.

BJPP also aims to participate effectively in the meetings of the Brazilian Association of Scientific Editors (ABEC). In 2012, two meetings were held with the presence of the Editor-in-Chief of BJPP. Such meetings enable editors of scientific journals to insert their publications into the discussed subjects, and by sharing experiences with other editors, many problems are effectively solved. And in the quality of Editor-inChief of BJPP, I'll sponsor the ABEC meeting in 2014, to be held in Campos dos Goytacazes, RJ.

Also in 2012, the issues of BJPP have gained more mobility. They are available for tablets (iPad and Android based tablets). Therefore, we invite all of you who are interested to download the app BJPP in order to explore all the issues of 2012, including the cover. Soon, we will make the whole collection available, since BJPP's foundation.

Ricardo Bressan-Smith Editor-In-Chief, BJPP 
